\title{
A General Statement of Structured Singular Value Concepts
}

\author{
James Krause Blaise Morton Dale Enns Gunter Stein John Doyle Andrew Packard \\ Honeywell Systems and Research Center *
}

\begin{abstract}
Some key concepts of structured singular value theory for the stability- and performance-robustness analysis of linear time-invariant multivariable systems are stated. Using a set-invariance principle, the theory is then generalized to allow for nonlinear and/or time-varying nominal systems and uncertainties. The general theory is then re-specialized to the case of nominally linear time-invariant systems subject to $L_{2}$-induced-norm bounded uncertainties.
\end{abstract}

\section{Introduction}

Structured singular value (SSV) theory for the robustness analysis of control systems is developed in ([3], [4], [5], [6]), using a linear time-invariant (LTI) framework for plant and uncertainty, and a frequency-domain representation of the system. In [2], a state space representation of the nominal LTI system is used in the structured singular value calculation, and the theory is extended to allow for nonlinear time-varying (NLTV) uncertainty.

This paper is different in two respects: (1) an operator representation of the system and uncertainty is used, rather than a frequency domain or state space representation; (2) a eertain set-invariance principle (explained later) is used in place of a commutativity principle of the LTI theory. The operator representation allows nominal systems which are not LTI, and the set-invariance principle allows uncertainty and so-called "D-scales" which are not LTI. These modifications lead readily to generalizations of key LTI structured singular value expressions and robustperformance interpretations.

A special case of the generalization is the LTI control of a nominally LTI plant, with NLTV uncertainty characterized by an induced- $L_{2}$ norm bound (as treated in [2]). We specialize our generalization to this case, obtaining an SSV expression which is computationally tractable. The calculation differs from the state-space approach of [2], and the frequency-domain approach of ([3], [4], [5]), yet is similar to each in some respects.

The paper is organized as follows. Selected results from the existing LTI structured singular value theory are stated briefly in Section 2. Section 3 states one possible generalization of the notion of robust stability to the nonlinear case. Results corresponding to those of Section 2

\footnotetext{
- Other affiliations: John Doyle and Andrew Packard, Caltech; Dale Enns, U of Minnesota; Gunter Stein, MTT.
}

are derived for the general NLTV case in Section 4. To allow the reader to follow the parallelism with Section 2, corresponding equations are numbered the same in Sections 2 and 4, with prefix "LTI" in Section 2 and the prefix "G" (for General) in Section 4. Section 5 contains a respecialization of the general theory to the case of nominally LTI systems and $L_{2}$-induced-norm bounded uncertainty. Again, the equations are numbered the same as their counterparts in Sections 2 and 4, but with a prefix "NBO" (for Norm Bounded Operator).

\section{Structured Singular Values for Linear Time Invari- ant Systems}

For future comparison purposes, we first state a few basic equations of the now common structured singular value theory for linear time-invariant systems (see [3], [4], [5] for details).

A system with uncertainty blocks at arbitrary locations, as well as a performance criterion, can always be re-drawn in the form of Figure 1 , where $M$ is a known stable LTI transfer function depending on the plant design model and the control system [4]. $\Delta$ represents the structured uncertainty. It is assumed that the structure of $\Delta$ is given by

$$
\begin{gathered}
\Delta \in X, \\
\text { where } X=\left\{\Delta \mid \Delta=\operatorname{diag}\left\{\Delta_{i}, i=1,2, \ldots, n\right\},\right. \\
\Delta_{i} \text { is linear time-invariant, } \\
\left.\left\|\Delta_{i}\right\|_{\infty} \leq 1\right\} .
\end{gathered}
$$

Here \|\|$_{\infty}$ denotes the $H^{\infty}$ norm.

For complex matrices $M$ and $\Delta$, the structured singular value is defined by

$\mu(M)=\{\min \varepsilon \mid \operatorname{det}(I-\epsilon \Delta M)=0 \text { for some } \Delta \in X\}^{-1}$

The following results appear in the literature:

$$
\mu(M) \leq \sup _{\omega} \bar{\sigma}(M(j \omega))
$$

where $\sigma$ denotes the maximum singular value.

$$
\begin{gathered}
\mu(M)=\mu\left(D M D^{-1}\right) \text { for } D \in \mathbf{D} \\
\text { where } \mathbf{D}=\left\{D \mid D \Delta D^{-1}=\Delta \forall \Delta \in X\right\} .
\end{gathered}
$$

For the uncertainty set $X$ defined by (LTI-2), one satisifies (LTI-6A) by choosing

$$
\mathbf{D}=\left\{D \mid D=\operatorname{diag}\left\{d_{i}(j \omega) I_{i}\right\}, \quad i=1,2, \ldots, n\right\}
$$


and where $d_{i}(j \omega)$ is a frequency varying complex number and $I_{i}$ is an identity matrix of the same dimension as $\Delta_{i}$. Equation (LT7-6B) follows from the commutativity property

$$
D(j \omega) \Delta(j \omega)=\Delta(j \omega) D(j \omega) \quad \forall D \in D, \Delta \in X .
$$

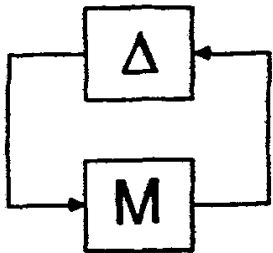

Figure 1

General Interconnection Structure

It follows that

$$
\mu(M) \leq \sup _{\omega}\left(\inf _{D \in D} \sigma\left(D M D^{-1}\right)\right) \text {. }
$$

Furthermore, for three or fewer $\Delta_{i}$ blocks, the above upper bound is actually an equality:

$$
\mu(M)=\sup _{\omega}\left(\inf _{D \in D} \sigma\left(D M D^{-1}\right)\right\}, \quad n \leq 3 .
$$

Equation (LT1-8) leads directly to efficient software for $\mu$ calculation.

When one $\Delta_{i}$ block is not actually an uncertainty, but is an added fictitious uncertainty representing a performance criterion, the interpretation of $\mu$ is

$$
\begin{gathered}
\mu(M)<1 \text { is necessary and sufficient } \\
\text { for robust performance. }
\end{gathered}
$$

More can be said about the LTI $\mu$ theory and software, but the above summarizes the essentials of the theory.

\section{Nonlinear System Robust Stability}

To state the structured singular value theory for nonlinear systems, it is necessary that we first select a notion of robust stability. A variety of options are possible.

Consider the system of Figure 2, with

$$
M=\left[\begin{array}{ll}
M & M_{12} \\
M_{21} & M_{22}
\end{array}\right]
$$

We assume that all four sub-blocks of $M$ have bounded gain, and that $\Delta$ has bounded gain (uniformly over all $\Delta \in X$ ). only if

We will say that the system is robustly stable if and

$$
\sup _{\Delta \in X}\left\|(I-M \Delta)^{-1}\right\|<\infty,
$$

where HII denotes the appropriate induced-norm on the operator. (Remark: The "best" choice of a norm in which to define stability is an open issue deserving study, but is outside the scope of this paper.)

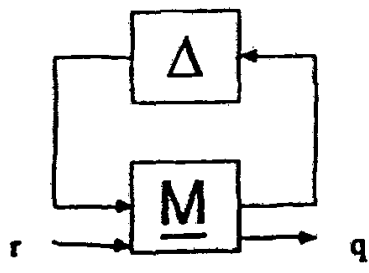

Figure 2

Interconnection with Inputs and Outputs

From the small gain theorem ([7], [1]), one finds that, when the system satisfies our robust stability definition.

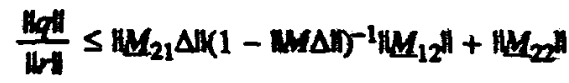

which is uniformly bounded over $\Delta \in X$. Thus we have adopted a bounded-gain notion of stability.

When the robust stability condition is not satisfied, it need not follow (as in the LTI case) that some $\Delta \in X$ yields unbounded gain from $r$ to $q$, due to the perversions possible with arbitrary nonlinear operators. Thus our notion of robust stability is that of guaranteed bounded gain using the small gain theorem.

Remark: one could formulate the condition in terms of $\Delta M$ in addition to, or instead of, $M \Delta$. For simplicity, we will use only the above definition.

The notion of robust performance we will adopt is the satisfication of the robust stability condition, and in addition

$$
\sup _{*} \sup _{\Delta \in X} \frac{\|q\|}{\|+\|}<1
$$

Note: non-unity performance requirements are supported; one simply normalizes the problem by absorbing weights on $q$ or $r$ into $M$, as in the references for the LTI case.

\section{Generalized Structured Singular Values}

To treat a broader class of problems, such as nonlinear and/or time-varying $M$ or $\Delta$, one must re-derive the $\mu$ theory in a generalized form, which we do briefly here.

Assume that the structure of $\Delta$ is given by

$$
\Delta \in X \text {, }
$$

where $X$ bas been normalized such that $\|\Delta\| \leq 1$ for all $\Delta \in X$ (normalizatons can be absorbed into the system $M$ ). Without any further assumptions on the nature of the set $X$ or the operator $M$, one can define the structured singular value by

$$
\mu(M)=\left(\sup _{\Delta \in X}|\Delta \|| I-M \Delta \text { is not invertible }\right)^{-1}
$$

For this definition, the small gain theorem $([7],[1])$ implies that

$$
\mu(M) \leq\|M\|
$$

Notation: let $D X \hat{D}^{-1}$ denote the set $\left(D \Delta \hat{D}^{-1} \mid \Delta \in X\right)$. 
Theorem 1:

$$
\mu(M)=\mu\left(\hat{D} M D^{-1}\right) \text { for }(D, \hat{D}\} \in \mathrm{D}
$$

where $D$ is any set of pairs of operators for which each pair $\{D, \hat{D}\}$ satisfies

$$
D X \hat{D}^{-1}=X \text {. }
$$

Note that the commutativity principle involved in (LTI-6) is not required. It is not required that each of the elements of the uncertainty set is invariant under the socalled D-scale operations, but only that the uncertainty set as a whole is invariant under the D-scale operations. This is the set-invariance principle alluded to in the introductory remarks.

An immediate consequence of (G-5) is

$$
\mu(M) \leq \inf _{\{D, \hat{D}\} \in \mathrm{D}}\left\|\hat{D} M D^{-1}\right\|
$$

Note that in the general version, $\Delta$ need not be LTI or square or block diagonal, $D$ need not commute with $\Delta$, and $M$ need not be LTI or square.

The theorem can be understood through examination of Figure 3. The system of Figure 3 is clearly the same system as Figure 1, so either both are stable or both are unstable. The subsystem above the dotted line is an uncertain operator, which takes on any value in $X$ (by the assumptions of the theorem). We thus have a new " $M^{\prime \prime}$, namely $M_{\text {new }}=\hat{D} M D^{-1}$, coupled with the same uncertainty set, which can be unstable if and only if the original system can be unstable. Writing down the definition of $\mu$ for the two systems, it is not surprising that $\mu$ is the same for both. This is fact is formally proved in the appendix.

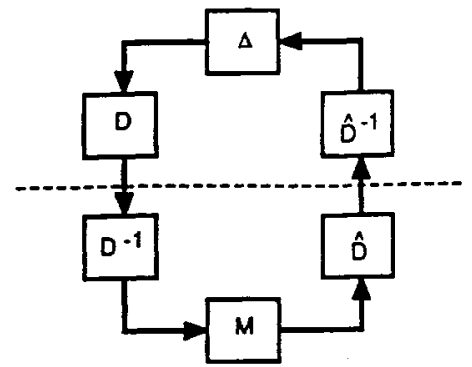

Figure 3

System of Theorem 1

Moving on to the question of robust performance, suppose an artificial $\Delta$ block $\Delta_{P}$ is added for performance. The remainder of the system can be denoted $F(\Delta)$, as shown in Figure 4. In the figure, " $\mathrm{I}$ " could represent the external excitations such as cormmands and disturbances. The signal "q" represents the output one does not want to become large - usually the command tracking error signal or the actuator outputs. To impose the performance requirement $\|F(\Delta)\| \leq 1 \forall \Delta \in X$, one chooses the characterization for the fictitious $\Delta_{p}$ to be $\left\|\Delta_{p}\right\| \leq 1$.

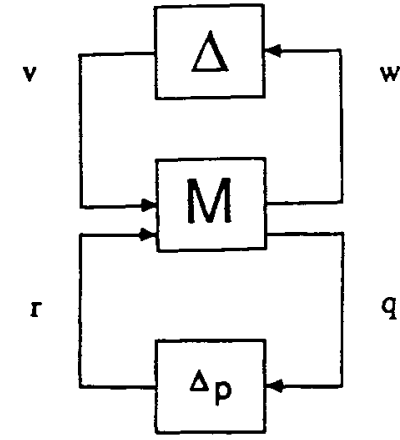

(B)

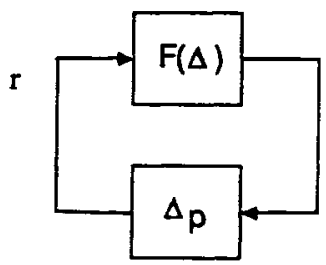

$q$

Figure 4

System for Performance Robustness Analysis

Note that the norms on the input and ouput spaces have been left arbitrary -- one can choose, for example, $L_{\infty}$ norms if these are what is of interest. Note also that the dimension of the input and ouput vectors are not specified, and $\Delta_{P}$ need not be square, allowing for a certain class of "multiple performance objectives" problems.

Theorem 2: $\mu(M)<1$ is necessary for robust performance. (Proved in appendix.)

Theorem 3:

$$
\{D, \hat{D}\} \in \mathrm{D}
$$

is sufficient for robust performance.

(Proved in appendix.)

It can be seen that (LTI-1), (LTI-3), (LTI-4), (LTI-5), and (LTI-6) are precisely special cases of the general cases (G1), (G3), (G4), (G5), and (G6). The LTI versions result from adding the specializing assumption LTI-2. A stronger interpretation of $\mu$ in the robust performance problem also results from the specializing assumption.

\section{Structured Singular Values for Norm Bounded Operator Uncertainty}

The general theory allows one to choose alternate assumptions which fit the practical needs of the problem. To illustrate this, we will now re-specialize the general theory to allow for nonlinear and time-varying (NLTV) uncertainties.

Consider the case in which the controller and design model are still LTI (i.e., $M$ is LTI), but replace the uncertainty assumption (LTI-2) with the norm-bounded operator (NBO) assumption:

$$
X=\left\{\Delta \mid \Delta=\operatorname{diag}\left\{\Delta_{i}, i=1,2, \ldots, n\right\},\left\|\Delta_{i}\right\|_{i 2} \leq 1\right\} .(\mathrm{NBO}-2)
$$


Here \|\|$_{12}$ is the induced $L_{2}$ norm. Since for linear $\Delta_{i}$ 's as in LTI-2, $\left\|\Delta_{i}\right\|_{\infty}=\left\|\Delta_{i}\right\|_{i 2}$, the new assumption (NBO-2) includes all of the same set of uncertainty as (LTI-2), as well as all nonlinear and/or time-varying uncertainty with the same induced 2-norm bound.

Theorem 4: Under assumption (NBO-2), (G-6) is satisfied by the set

$$
\mathbf{D}=\left\{(D, \hat{D}\} \mid D=\operatorname{diag}\left\{d_{t} f_{i}\right\}, \hat{D}=\operatorname{diag}\left\{d_{i} \hat{I}_{i}\right\}\right.
$$

where $d_{i}$ is a constant complex number and $I_{i}, \hat{I}_{i}$ are identities with dimensions given respectively by the number of rows and columns of $\Delta_{i}$. (Proved in appendix.)

$$
\begin{aligned}
& \text { (G4) and (G5) yield } \\
& \qquad \mu(M) \leq \inf _{D \in D}\left\|D M D^{-1}\right\| .
\end{aligned}
$$

Since $D$ is linear,

$$
\mu(M) \leq \inf _{D \in D}\left\{\sup _{\omega} \sigma\left(D M D^{-1}\right)\right\} .
$$

While (NBO-7) may resemble (LTI-7), it is not the same since the order of the "inf" and "sup" cannot be reversed, and since the set $D$ is different. These distinctions are very important in calculation of $\mu$; one obtains altogether different results. As an example of the difference between (LTI-7) and (NBO-7), consider

$$
M=\left[\begin{array}{cc} 
& \frac{10 s^{2}}{.9} \\
\frac{10}{(s+100)^{2}} \\
.9
\end{array}\right]
$$

Let $\Delta=\operatorname{diag}\left\{\Delta_{1}, \Delta_{2}\right\}$. If we assume $\Delta_{i}$ are LTI, equation (LTI-7) yields $\mu_{L T I} \approx 0.999$. Assuming $\Delta_{i}$ are norm bounded possibly NLTV operators, (NBO-7) yields $\mu_{N B O} \approx 10$. The reason for this difference is that frequency-dependent $D$ 's can be used in the LTI case but not in the NLTV case.

The difference in the two calculations has considerable practical significance. If $\boldsymbol{M}$ represented a nominal closed-loop control system design corrupted by uncertainty, one would conclude that the control system was robust to linear perturbations of the specified size, but very nonrobust to nonlinear time-varying perturbations of the same "size."

\section{Conclusion}

Key structured singular value concepts for linear time-invariant systems generalize easily to nonlinear timevarying systems through the use of a set-invariance principle in place of a commutativity property, and an operator representation of the nominal system and the uncertainty. The generalization includes, as special cases, both the previous LTI results of [3], [4], [5], and the nominally-linearwith-NLTV-uncertainty results of [2]. In the latter case, the computations involved with our approach are somewhat different from those of the state-space approach of [2].

\section{Acknowledgment}

The authors have relied extensively on the work of John Doyle.

\section{APPENDIX}

Proof of Theorem 1: First note that $D^{-1} X \hat{D}=X$ since, by the hypothesis of the Theorem, $X=D X \hat{D}^{-1}$ hence $D^{-1} X \hat{D}=D^{-1} D X \hat{D}^{-1} \hat{D}=X$.

Now, for invertible $\hat{D}$,

$I-\hat{D} M D^{-1} \Delta$ not invertible $\Longleftrightarrow I-M D^{-1} \Delta \hat{D}$ not invertible (this follows through multiplication on the left by $\hat{D}^{-1}$ and on the right by $\hat{D})$. Now since $D^{-1} X \hat{D}=X$, it follows from the definition of $\mu$ that $\mu\left(\hat{D} M D^{-1}\right)=\mu(M)$.

Proof of Theorem 2: First note that Figure 4 indicates the method of performance robustness analysis. In the figure, $\Delta$ represents the system uncertainty, and $\Delta_{P}$ is a fictitious uncertainty block added to obtain robust performance. The reason this works is that the system of Figure 4 can be redrawn as the system of Figure $4 \mathrm{~B}$, and the question of stability with $\Delta_{P}$ present is related to the gain of the operator $F(\Delta)$. While this has been established for the case of linear systems, here we establish the necessity of $\mu<1$ to achieve $\|F(\Delta)\|<1$ for all allowable $\Delta$ (robust performance).

The proof that $\mu<1$ is necessary for robust performance, is by contradiction. Assume that $\mu>1$ and $\|F(\Delta)\|<1$ for all $\Delta \in X . \mu>1$ implies that the system is singular for some $\hat{\Delta} \in X$ and $\hat{\Delta}_{P}$ of norm less than one.

Case 1: $F(\hat{\Delta})$ is unstable. Then clearly robust performance is contradicted.

Case 2: $F(\hat{\Delta})$ is stable. Since $\hat{\Delta}$ and $\hat{\Delta}_{p}$ destabilize the system of Figure $4 B$, the small gain theorem of Zames [7] can be used to show $\left\|\hat{\Delta}_{p}\right\|\|F(\hat{\Delta})\| \geq 1$ therefore $\|F(\hat{\Delta})\| \geq 1$, contradicting robust performance.

Hence theorem 2.

Proof of Theorem 3: In practice, the choice of norms for the signals $v$ and $w$ of Figure 4 would be determined by the nature of the uncertainty. The choice of norms for $q$ and $r$ would be determined by the nature of the performance objectives. However, when combining the two outputs, we are free to define the net norm on $\left[\begin{array}{l}w \\ q\end{array}\right]$ in a variety of ways. Thus we may let the induced norm on $M$ of Figure 4 correspond to input and output norms of the form:

$$
\begin{aligned}
& \mid \begin{array}{l}
v \\
|r|_{\text {in }}
\end{array}:=\|v\|_{v}+\|r\|_{r} \\
& \mid \begin{array}{l}
w \\
|\boldsymbol{q}|_{\text {out }}
\end{array}:=\|w\|_{w}+\|q\|_{q}
\end{aligned}
$$

where the subscript to the norm indicates that the various norms may be of different types, depending on the nature of the uncertainty or the performance objective. By a change of notation, one may use " $M^{n}$ in place of "D́DD ${ }^{-1 n}$, with the hypothesis rewritten as $\|M\|<1$. That is,

$$
\|w\|_{w}+\|q\|_{q} \leq\|v\|_{v}+\| H_{r}
$$

By the normalization of the uncertainy set $X$,

$$
\|v\|_{v} \leq\|w\|_{w} \text {. }
$$


Adding the above inequalities,

$$
\|v\|_{v}+\|w\|_{w}+\|q\|_{q} \leq\|v\|_{v}+\|w\|_{w}+\|r\|_{r}
$$

Therefore

$$
\|q\|_{q} \leq\|r\|_{r}
$$

regardless of the value in $X$ assumed by $\Delta$. Thus $F(\Delta)$ in Figure $4 B$ has norm less than one for all $\Delta \in X$, which is the desired robust performance result.

Lemma 2: Let $B_{1}$ and $B_{2}$ be Banach spaces. Consider the sets of operators

$$
X=\left\{x: B_{1} \rightarrow B_{2}\|x\| \leq 1\right\}
$$

$$
\begin{array}{r}
\mathbf{D}=\left\{\{D, \hat{D}\} \mid D: B_{2} \rightarrow B_{2}, \frac{\|D v\|}{\|v\|}=\frac{\|D u\|}{\|u\|}=d \forall u, v \in B_{2},\right. \\
\left.\hat{D}: B_{1} \rightarrow B_{1}, \frac{\|\hat{D} v\|}{\|v\|}=\frac{\|\hat{D} u\|}{\|u\|}=d \forall u, v \in B_{1}\right\}
\end{array}
$$

Proposition of Lemma 2: $D X \hat{D}^{-1}=X$.

Proof of Lemma 2: Suppose $x \in X$ and $v \in B_{1}$. Then $\left\|\hat{D}^{-1} v\right\|=d^{-1}\|v\|,\left\|x \hat{D}^{-1} v\right\| \leq d^{-1}\|v\|,\left\|D x \hat{D}^{-1} v\right\| \leq\|v\|$. Thus $x \in X$ implies $D x \hat{D}^{-1} \in X$.

Now suppose $D x \hat{D}^{-1} \in X$. Then $\left\|D x \hat{D}^{-1} v\right\| \leq\|v\|$. Let $q=\hat{D}^{-1} v$. Then $\|v\|=d\|q\|$ and $\left\|D x \hat{D}^{-1} v\right\|=d\|x q\|$. Substitution yields dllxq\| $\leq$ dlall. Since $v$ is arbitrary and all $q$ can be reached by $\hat{D}^{-1} v,\|x\|<=1$. Thus $D x \hat{D}^{-1} \in X$ implies $\boldsymbol{x} \in \boldsymbol{X}$.

Proof of Theorem 4: By the choice of dimensions of the $I_{i}$ and $\hat{I}_{i}$,

$$
D \Delta \hat{D}^{-1}=\operatorname{diag}\left\{d_{i} \Delta_{i} d_{i}^{-1}\right\}
$$

Now Theorem 4 is a special case of Lemma 2.

Remark: Lemma 2 actually characterizes the largest set D which works for the set of norm-bounded operators. Lemma 2 implies that, in Theorem 4, one can let $D=\operatorname{diag}\left\{d_{i} U_{i}\right\}$ where $U_{i}$ is any unitary operator.

\section{References}

1. C. A. Desoer and M. Vidyasagar, Feedback Systems: Input-Output Properties, Academic Press, 1975.

2. J. Doyle and A. Packard, "Uncertain Multivariable Systems from a State Space Perspective," Proceedings of the American Control Conference, Minneapolis, 1987.

3. J. C. Doyle, "Analysis of Feedback Systems with Structured Uncertainty," IEE Proceedings, Part $D$, vol. vol. 129, No. 6, November, 1982.

4. J. C. Doyle, J. E. Wall, and G. Stein, "Performance and Robustness Analysis for Structured Uncertainty," Proceedings of the 20th Conference on Decision and Control, December, 1982.

5. J. C. Doyle, "Structured Uncertainty in Control System Design," Proceedings of the 24th Conference on Decision and Control, Ft. Lauderdale, Florida, December, 1985.

6. M. Fan and A. Tits, "Characterization and Efficient Computation of the Structured Singular Value," IEEE Transactions on Automatic Control, vol. AC-31, August 1986.

7. G. Zames, "On the Input-Output Stability of Nonlinear Time-Varying Feedback Systems, Part I and II," IEEE Transactions on Automatic Control, vol. AC-11, 1966. 